\title{
Bowel Preparation, the First Step for a Good Quality Colonoscopy
}

\author{
Ho-Su Lee, Jeong-Sik Byeon \\ Department of Gastroenterology, Asan Medical Center, University of Ulsan College of Medicine, Seoul, Korea
}

\section{Article: Comparison of the Efficacy and Tolerability between Same-day Picosulfate and Split- dose Polyethylene Glycol Bowel Preparation for Afternoon Colonoscopy: A Prospective, Randomized, Investigator-blinded Trial (Intest Res 2014;12:53-59)}

Colorectal cancer (CRC) is the third most common cancer and the third leading cause of cancer death in Western countries. ${ }^{1}$ In addition, the incidence of and mortality from CRC are increasing in Asian countries. ${ }^{2,3}$ CRC has become one of important worldwide public health issues. Therefore CRC screening in asymptomatic subjects is crucial for the reduction of incidence of CRC and colonoscopy is an important screening modality. ${ }^{4}$

In an effort to further lower CRC mortality, many approaches to quality improvement in colonoscopy have been proposed. They include adequate withdrawal time, optimal withdrawal technique, cecal intubation rate, optical enhancement and measurement of adenoma detection rate. In addition to these various quality indicators, the success of colonoscopy further depends on the quality of bowel preparation. Suboptimal bowel preparation is associated with a longer cecal intubation time, a lower cecal intubation rate, and a lower polyp detection rate. ${ }^{5}$ They also lead to increased costs associated with colonoscopy because suboptimal colonoscopy often necessitates more frequent, shorter screening and surveillance intervals than recommended. ${ }^{6}$ It remains to be seen what is the best method of bowel preparation for a good quality colonoscopy. An ideal bowel preparation should be small in volume, acceptable and tolerable to patients, reliable in cleansing efficacy, and safe. ${ }^{7}$

Received November 18, 2013. Revised November 20, 2013.

Accepted November 21, 2013.

Correspondence to Jeong-Sik Byeon, Department of Gastroenterology, Asan Medical Center, University of Ulsan College of Medicine, 88 Olympic-ro 43gil, Songpa-gu, Seoul 138-736, Korea. Tel: +82-2-3010-3180, Fax: +82-2476-0824,E-mail: jsbyeon@amc.seoul.kr

Financial support: None. Conflict of interest: None.
In this issue of Intestinal research, Kang et al. investigate the efficacy and tolerability of bowel preparation for afternoon colonoscopy. ${ }^{8}$ Study subjects were randomized to one of two therapeutic dosing strategies: the same-day two sachets of picosulfate group ( $n=97$, group A) vs. conventional split-dose 4 L polyethylene glycol (PEG) group ( $n=99$, group B). The primary endpoint was bowel preparation success assessed by using the Ottawa scale. Patients' tolerability was assessed using a questionnaire immediately before colonoscopy to evaluate patient tolerability, adverse events, and sleep quantity. The number of polyps and adenomas detected were measured.

The Ottawa score of the total colon was $4.05 \pm 1.56$ in the group A and $3.80 \pm 1.55$ in the group B $(P=0.255)$. Bowel preparation success was achieved in $61.5 \%$ of the same-day picosulfate group vs. $71.3 \%$ of $4 \mathrm{~L}$ PEG group, which failed to reach statistical significance $(P=0.133)$. However, tolerability of the group A regimen was superior to the group B regimen $(P<0.001)$. The overall incidence of adverse events was fewer in the same-day picosulfate group $(P=0.037)$. In addition, the proportion of patients who slept at least $80 \%$ of their usual sleeping hours was higher in group A than group B (87.8 vs. $56.4 \%, P<0.001)$. Adenoma detection rate was similar between the two groups (31.3\% for group A $v s .34 .3 \%$ for group $\mathrm{B} ; P=0.651)$. These results suggest that bowel preparation with the same-day picosulfate regimen may be similar in cleansing efficacy and superior in the tolerability and adverse event profile to the conventional split-dose $4 \mathrm{~L}$ PEG regimen.

The conclusion of this study may have several implications. First, safety is an important virtue. Therefore, safer adverse event profile of the same-day picosulfate regimen is meaningful by itself. Second, shortening of bowel preparation duration within a single day will less severely disturb a

( ) Copyright 2014. Korean Association for the Study of Intestinal Diseases. All rights reserved.

This is an Open Access article distributed under the terms of the Creative Commons Attribution Non-Commercial License (http://creativecommons.org/licenses/by-nc/3.0)

which permits unrestricted non-commercial use, distribution, and reproduction in any medium, provided the original work is properly cited. 
patient's daily life, which may be beneficial in the viewpoint of cost-effectiveness such as economic and social activity of the patient. Third, experience of difficult bowel preparation may lead to the poorer compliance for the next colonoscopy such as surveillance after initial polypectomy, which makes colonoscopy screening and surveillance program ineffective for adequate CRC prevention. In this regard, more tolerable bowel preparation regimen may be better in terms of optimal CRC prevention by improving compliance for repeated colonoscopy examinations.

Despite these positive implications of the same-day picosulfate regimen in this study, there are several issues to be addressed before the conclusion and implications would be completely admitted. First, as the authors mentioned, this study did not analyze the biochemical profile including the electrolyte and renal function before and after bowel preparation. Because of the potential possibility of hyponatremia, dehydration and other electrolyte imbalance after bowel cleansing with laxative, meticulous evaluation of biochemical profile should be performed, especially in the high risk population such as elderly patients and those with congestive heart failure, cirrhosis, or kidney disease. Second, it should be noted that the score of the right colon was $1.65 \pm 0.70$ in the same-day picosulfate group and $1.43 \pm 0.77$ in the $4 \mathrm{~L}$ PEG group $(P=0.046)$. Without adequate right colon preparation, one can miss more right colon polyps. Although overall adenoma detection rate was not different between the two preparation regimen, we do not know the adenoma detection rate according to the location in colorectum. Third, this study did not analyze the adenoma detection according to the morphology and histology of polyps. Considering the fact that sessile serrated adenoma/polyp which is usually flat is common in the right colon and the result that the right colon preparation was poorer in the same-day picosulfate group in this study, certain types of polyps in the right colon may have been missed more commonly in the same-day picosulfate group. Because right colon is associated more frequently with the interval cancer, ${ }^{9}$ this assumption may be clinically important. Therefore, improvement of right colon preparation quality with the same-day picosulfate regimen and analysis of adenoma detection according to the morphology and histology should be performed.

There are several bowel cleansing regimen for colonoscopy. However, most of them are not completely satisfactory in terms of efficacy, safety and tolerability. Therefore, new regimen should be developed and investigated for better bowel preparation. The study by Kang et al. in this issue of Intestinal research analyzed the clinical usefulness of the same-day picosulfate regimen, which enlarged the armamentarium for bowel preparation. Further studies are warranted to confirm the usefulness of this regimen and to clarify methods to improve the quality of bowel preparation by this regimen. Investigations for better bowel preparation may ultimately lead to the more effective CRC screening and surveillance and decreased CRC incidence and mortality.

\section{REFERENCES}

1. Siegel R, Naishadham D, Jemal A. Cancer statistics, 2013. CA Cancer J Clin 2013;63:11-30.

2. Byeon JS, Yang SK, Kim TI, et al. Colorectal neoplasm in asymptomatic Asians: a prospective multinational multicenter colonoscopy survey. Gastrointest Endosc 2007;65:1015-1022.

3. Sung JJ, Lau JY, Goh KL, Leung WK. Increasing incidence of colorectal cancer in Asia: implications for screening. Lancet Oncol 2005;6:871-876.

4. Winawer SJ, Zauber AG, Ho MN, et al. Prevention of colorectal cancer by colonoscopic polypectomy. The National Polyp Study Workgroup. N Engl J Med 1993;329:1977-1981.

5. Froehlich F, Wietlisbach V, Gonvers JJ, Burnand B, Vader JP. Impact of colonic cleansing on quality and diagnostic yield of colonoscopy: the European Panel of Appropriateness of Gastrointestinal Endoscopy European multicenter study. Gastrointest Endosc 2005;61:378-384.

6. Rex DK, Imperiale TF, Latinovich DR, Bratcher LL. Impact of bowel preparation on efficiency and cost of colonoscopy. Am J Gastroenterol 2002;97:1696-1700.

7. Brown AR, DiPalma JA. Bowel preparation for gastrointestinal procedures. Curr Gastroenterol Rep 2004;6:395-401.

8. Kang MS, Kim TO, Seo EH, et al. Comparison of the efficacy and tolerability between same-day picosulfate and split-dose polyethylene glycol bowel preparation for afternoon colonoscopy: a prospective, randomized, investigator-blinded trial. Intest Res 2014;12:53-59.

9. Brenner H, Chang-Claude J, Seiler CM, Hoffmeister M. Interval cancers after negative colonoscopy: population-based casecontrol study. Gut 2012;61:1576-1582. 